\title{
ORIGINAL ARTICLE Reduced FOV diffusion tensor MR imaging and fiber tractography of pediatric cervical spinal cord injury
}

\author{
M Alizadeh ${ }^{1,2,3}$, A Intintolo ${ }^{1,2}$, DM Middleton ${ }^{1,2,3}$, CJ Conklin²,3,4, SH Faro ${ }^{1,2,4}$, MJ Mulcahey ${ }^{5}$ and \\ FB Mohamed ${ }^{1,3,4}$
}

Study design: Quantitative study.

Objectives: To evaluate the effectiveness of pediatric spinal cord diffusion tensor tractography (DTT) generated from reduced field of view diffusion tensor imaging (DTI) data and investigate whether there are differences in these values between typically developing (TD) subjects and patients with spinal cord injury (SCI).

Setting: Temple University Hospital and Shriners Hospitals for Children-Philadelphia, USA.

Methods: A total of 20 pediatric subjects including 10 healthy subjects (age $15.13 \pm 3.51$ years (mean \pm s.d.) and age range 11-21 years) and 10 subjects with $\mathrm{SCl}$ in the cervical area (age 13.8 \pm 3.26 years and age range 8-20 years) were recruited, and scanned using a 3.0T MR scanner. Quantitative parameters of DTI and fiber tracking, such as mean fractional anisotropy (FA), apparent diffusion coefficient (ADC), mean length of fiber tracts and tract density, were calculated for each subject.

Results: Subjects with SCl showed reduced FA and tract density, and increased ADC values and length of fiber tracts, compared with controls. Statistically significant differences were seen in FA $(P=0.0238)$ and tract density $(P=0.0005)$ between controls and subjects with $\mathrm{SCl}$, whereas there were no significant differences in ADC values and length of fiber tracts. The tractography visually showed that the white matter tracts (blue color) of the $\mathrm{SCl}$ patients were overall less abundant and less organized compared with control cases.

Conclusion: The results show that DTI and DTT could be used as surrogate markers for quantification and visualization of the injured spinal cord.

Spinal Cord (2017) 55, 314-320; doi:10.1038/sc.2016.121; published online 16 August 2016

\section{INTRODUCTION}

Magnetic resonance imaging (MRI) is an essential tool for detecting pathological changes, for predicting prognosis and for planning the treatment of patients with spinal cord injury (SCI). ${ }^{1}$ However, the information typically provided by conventional T1- and T2-weighted MRI of the spinal cord is limited to the differentiation of white matter from gray matter and macroscopic structural changes within the cord in a diseased or injured state. ${ }^{2}$ Conventional MRI depicts the white matter as a uniform tissue, despite it being composed of a complex array of directionally oriented nerve fibers. Methods to visualize the pathways of the white matter in vivo have been long sought, and diffusion tensor tractography (DTT) has demonstrated this ability. ${ }^{1,2}$

Diffusion tensor imaging (DTI) is a technique that allows quantification of the diffusion of water molecules in each voxel of an image in directions parallel and transverse to the plane of neuronal axons. ${ }^{3,4}$ The quantitative characteristic of DTI allows the characterization of physical properties of tissues. The highly directional architecture of the spinal cord may allow DTI to accurately localize white matter, separate white from gray matter, and assess structural damage of the cord by modeling the direction and magnitude of water diffusion. This information enables DTI to encode the direction and magnitude of water diffusion, allowing for inference of the orientation of white matter fibers on a voxel-by-voxel basis. ${ }^{3-6}$

Tractography algorithms utilize the DTI-encoded information to reconstruct white matter tracts. ${ }^{7}$ Tractography algorithms can be broadly divided into two categories: deterministic and probabilistic. Fiber assignment by continuous tracking (FACT) method is a streamline-based deterministic method that traces pathways from a seed region by following the primary eigenvector from one voxel to the next. ${ }^{2,7}$ It is a well-established and straightforward technique, which is able to generate high-resolution images of axonal projections. In the FACT technique, seeds are placed in voxels with fractional anisotropy (FA) greater than the predefined threshold (for example, 0.15 ) to include only white matter voxels and then grown in both directions along the dominant diffusion orientation into fiber tracts or streamlines. A tract is terminated when it reaches a voxel with sub-threshold FA, or when the turning angle exceeds some threshold (for example, $\left.70^{\circ}\right){ }^{8}$ The probabilistic algorithm defines pathways by generating multiple curves from seed points using a Monte Carlo simulation. Probability of connectivity is then assigned to individual voxels based on the frequency with which the curves traverse the voxels. In both deterministic and probabilistic techniques, prior

${ }^{1}$ Department of Bioengineering, Temple University, Philadelphia, PA, USA; ${ }^{2}$ Department of Radiology, Temple University, Philadelphia, PA, USA; ${ }^{3}$ Department of Radiology, Thomas Jefferson University, Philadelphia, PA, USA; ${ }^{4}$ Department of Electrical Engineering, Temple University, Philadelphia, PA, USA and ${ }^{5}$ Department of Occupational Therapy, Thomas Jefferson University, Philadelphia, PA, USA

Correspondence: M Alizadeh, Department of Bioengineering, Temple University, 909 Walnut Street, Philadelphia, PA 19107, USA.

E-mail: tuf07723@temple.edu

Received 30 November 2015; revised 31 May 2016; accepted 1 July 2016; published online 16 August 2016 
information of the anatomy of fiber tracts is needed to distinguish fiber tracts of interest and streamline tracts. ${ }^{8,9}$

The white matter of the spinal cord is composed of nerve fibers entering from the dorsal roots, nerve fibers exiting to ventral roots, and fiber bundles that travel in a craniocaudal direction in a relatively orderly manner. Although spinal tracts are anatomically similar, functionally each tract is distinct as they convey particular information from or toward the brain. Water within them diffuses along the course of the axons. ${ }^{10}$ These fibers can be tracked and visualized using DTT. ${ }^{11}$ To create DTT images, parameters such as the apparent diffusion coefficient (ADC), which is an expression of the magnitude of diffusion, and FA, which is representative of the anisotropy, or directionality, of the diffusion, must be calculated. The FA index is appropriately normalized so that it takes values from zero (when diffusion is isotropic) to one (when diffusion is constrained along one axis only). ${ }^{2,11}$

DTT reveals the course of fibers and allows in-vivo mapping of the diffusional properties of water molecules, which has the high degree of diffusional anisotropy in white matter. It tracks the direction of the major axis of the ellipsoid of every voxel and provides reconstruction of neuronal projections by tracking these vectors. ${ }^{12,13}$ DTT is a potential modality for performing in vivo tracking of axonal fibers. ${ }^{8,13}$ The properties and clinical applications of DTT in the brain have been widely reported, but technical difficulties (physiological motion, low signal to noise ratio (SNR), small size of cord) so far have limited DTT studies of the spinal cord, especially in the pediatric population (http://trackvis.org/blog/tag/diffusion-toolkit/). ${ }^{14}$ In this study, we report the effective use of DTT to visualize fiber tracts of the pediatric spinal cord in both normal individuals and SCI patients using DTI data collected with a reduced field of view (rFOV) spatially selective 2D radio frequency (RF) DTI pulse sequence. ${ }^{5}$ Data collection using this sequence has been shown to produce high-resolution reproducible images of the spinal cord within minimal scan time while mitigating artifacts. The tracts were reconstructed from the DTI data set acquired using this pulse sequence based on the deterministic FACT algorithm, executed using TrackVis 0.5 .2 and Diffusion Toolkit 0.6.2 software tools (http://trackvis.org/blog/tag/diffusion-toolkit/).

The purpose of this study was to (a) evaluate the feasibility of generating DTT images of the cervical spinal cord in both children with typical development and those with SCI using a deterministic method-namely, FACT, (b) to measure the DTI indices as well as tract-specific information using regions of interest (ROI) generated at

Table 1 Demographic and clinical characteristics of the $\mathrm{SCl}$ patients

\begin{tabular}{|c|c|c|c|c|c|}
\hline Subject & $\begin{array}{c}\text { Age at the } \\
\text { time of } \\
\text { study }\end{array}$ & $\begin{array}{c}\text { Age at the } \\
\text { time of } \\
\text { SCl }\end{array}$ & $\begin{array}{c}\text { Level of } \\
\text { injury based } \\
\text { on MRI }\end{array}$ & $\begin{array}{l}\text { Level of injury } \\
\text { based ISNCSCI }\end{array}$ & $\begin{array}{c}\text { Severity of } \\
\text { injury based } \\
\text { AIS }\end{array}$ \\
\hline $\mathrm{SCl} 1$ & 13 & 12 & $\mathrm{C} 1$ & $\mathrm{C} 1$ & B \\
\hline $\mathrm{SCl} 2$ & 13 & 11 & C5 & C5 & A \\
\hline $\mathrm{SCl} 3$ & 13 & 12 & $\mathrm{C} 4$ & C6 & C \\
\hline $\mathrm{SCl} 4$ & 20 & 16 & $\mathrm{C} 2$ & $\mathrm{C} 1$ & D \\
\hline $\mathrm{SCl} 5$ & 8 & 0.06 & $\mathrm{C} 4$ & $\mathrm{C} 8$ & B \\
\hline SCl6 & 17 & 13 & $\mathrm{C} 4$ & C5 & B \\
\hline $\mathrm{SCl} 7$ & 13 & 2 & $\mathrm{C} 4$ & C5 & $A$ \\
\hline $\mathrm{SCl} 8$ & 15 & 2 & $\mathrm{C} 4$ & $\mathrm{C7}$ & $A$ \\
\hline SCl9 & 11 & 0.04 & C3 & $\mathrm{C} 8$ & C \\
\hline SCl10 & 15 & 4 & $\mathrm{C} 2$ & C7 & B \\
\hline
\end{tabular}

Abbreviations: AIS, American Spinal Injury Association Impairment Scale; ISNCSCI, International Standards for Neurological Classification of Spinal Cord Injury; MRI, magnetic resonance imaging; $\mathrm{SCl}$, spinal cord injury. every axial slice location along the entire cervical spinal cord based on DTT images, and (c) to investigate whether there are differences in these values between the typically developing (TD) subjects and the patient group with SCI.

\section{MATERIALS AND METHODS}

\section{Subjects}

A total of 20 pediatric subjects including 10 healthy subjects (age $15.13 \pm 3.51$ years (mean \pm s.d.) and age range 11-21 years) who had no evidence of spinal cord injury or pathology and 10 subjects with SCI in the cervical area (age 13.8 \pm 3.26 years (mean \pm s.d.) and age range 8-20 years) were recruited. Subjects and parents provided written informed assent and consent for the institutional review board-approved protocol. As per the inclusion criteria used for recruitment of the spinal cord injury group, subjects had to have stable cervical-level spinal cord injury as evidenced by no neurological change in the past three months and had to be at least 6 months post cervical spinal cord injury. All patients were clinically assessed using the motor and sensory examinations of the International Standards for Neurological Classification of Spinal Cord Injury (ISNCSCI) $;{ }^{15}$ severity of injury was determined according the American Spinal Injury Association Impairment Scale (AIS). ${ }^{15}$ Of the subjects with cervical SCI (Table 1), there were three patients classified as AIS A (complete spinal cord injury), four with AIS B (sensory incomplete), two with AIS C (sensory and gravity-eliminated motor incomplete) and one with AIS A $\mathrm{D}$ (sensory and against gravity motor incomplete).

\section{Imaging}

The MRI scans were performed using a 3.0T Siemens Verio MR scanner (Siemens Healthcare, Erlangen, Germany) with 4-channel neck matrix and 8-channel spine array. The protocol consisted of a conventional sagittal turbo spin echo T1-weighted scan, a sagittal turbo spin echo T2-weighted scan, axial T2-weighted gradient echo and axial DTI scans based on the rFOV sequence described as follows. DTI images were acquired axially in the same anatomical location prescribed for the T2-weighted images to cover the entire cervical spinal cord (C1-C7 levels). ${ }^{5}$ The T2-weighted imaging parameters used were voxel size $=0.625 \times 0.625 \times 3.0 \mathrm{~mm}^{3}$, matrix size $=320 \times 320, \quad T R=3.6 \mathrm{~s}$, $\mathrm{TE}=90 \mathrm{~ms}$, slice thickness $=3 \mathrm{~mm}$, flip angle $=150^{\circ}$, number of averages $=1$ and acquisition time $=2.7 \mathrm{~min}$, and the DTI parameters used were number of directions $=20, \quad b=1000 \mathrm{~s} \mathrm{~mm}^{-2}$, voxel size $=1.2 \times 1.2 \times 3.0 \mathrm{~mm}^{3}$, matrix size $=36 \times 208$, axial slices $=35-45$ (depending on the subject's height), $\mathrm{TR}=6100-8000 \mathrm{~ms}, \mathrm{TE}=115 \mathrm{~ms}$, number of averages $=3$ and acquisition time $=7 \mathrm{~min}$. Cardiac gating and respiratory compensation were not used in this study as this will increase the acquisition time, which is not desirable in pediatric imaging. Also, anesthesia was not administrated to the subjects in this study.

\section{DTI acquisition using reduced-field-of-view sequence}

The rFOV sequence used in this study was a single-shot EPI sequence for diffusion-weighted imaging with spatially $2 \mathrm{D}-\mathrm{RF}$ excitations. It reduces geometric distortions due to magnetic field inhomogeneities as well as susceptibility differences between adjacent anatomical structures, and allows for higher in-plane resolutions. ${ }^{5,16}$ To optimize the rFOV sequence for imaging of the pediatric spinal cord, several diffusion directions $(6,12,20$ and 30), multiple signal intensity averages (1-4) and different $b$-values $(0,700,800,900$, 1000 and $1200 \mathrm{~s} \mathrm{~mm}^{-2}$ ) were examined. The final parameters (number of directions $=20, b=1000 \mathrm{~s} \mathrm{~mm}^{-2}$, and number of averages $=3$ ) were selected to ensure the relatively short acquisition time tolerable for pediatric imaging while maintaining high-spatial resolution that provides good accuracy for the estimate of the diffusion tensor.

\section{Post-processing of DTI data}

Motion artifacts induced by patient movement, spinal cord pulsation and oscillation, and cardiac and respiratory motion can be reduced by sedation, cardiac gating and respiratory compensation. Unfortunately, in pediatric imaging, these techniques increase the scanning time, which may lead to impatience in and lack of cooperation by the patient. ${ }^{6,17}$ To help mitigate these 


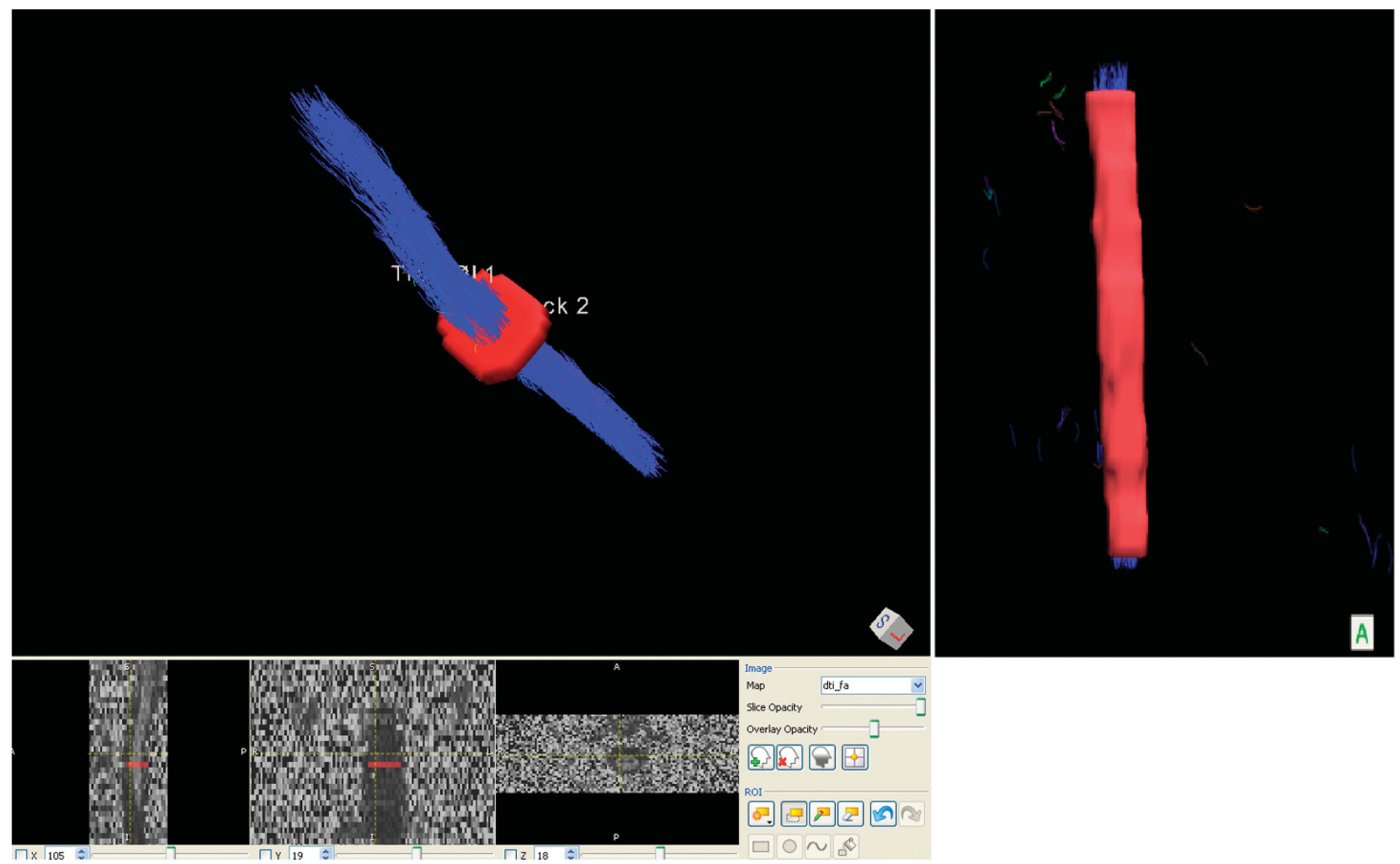

Figure 1 Illustration of placement of ROls drawn by an independent board-certified neuroradiologist for measuring DTI and DTT parameters using TrackVis software in a healthy subject.

effects, post-processing of the DTI was used to correct for motion artifacts. The Automated-Image-Registration (AIR) package implemented in DTIStudio software (www.mristudio.org) was used for this purpose. Diffusion directional images were aligned with the reference image $(\mathrm{B} 0)$ using a rigid registration algorithm and a scaled least square cost function., ${ }^{5,6}$

\section{Tensor estimation and fiber tracking}

After motion correction, diffusion tensor estimation and tractography were computed using Diffusion Toolkit software (http://trackvis.org/dtk/) with the scan-specific gradient table and image orientation information. The visualization program TrackVis (http://trackvis.org/) was used to visualize and analyze the tracts. Various DTI and DTT indices-namely, FA, ADC, tract length and tract density-were calculated.

\section{ROI definition}

Once the axial DTI images were acquired and DTT images created, the diffusion indices (FA and $\mathrm{ADC}$ ) were acquired by drawing circular ROIs on the axial FA maps at every axial level after being anatomically localized by an independent board-certified neuroradiologist. These ROIs were drawn including both the gray and white matters of the spinal cord. The ROIs excluded the border or edge of the cord ( 1-2 voxels from the outer margin of the cord) to avoid the effects of partial volume artifacts on the results that occur at the cord/cerebrospinal fluid (CSF) interface. This procedure was followed throughout all slices for all subjects, both healthy subjects and patients with SCI. Diffusion tensors at each pixel were calculated using multivariate linear least square fitting.

DTT images were generated by using a number of predefined tracking parameters. These are criteria determining where to place the seed and when to terminate a tract. Seeding can be defined with respect to any available image contrast using simple signal value thresholding. Tract termination can be based on parameter map values, much like seeding, but can also include tracking angle restrictions. Use of the FA map thresholding is an effective way to seed the regions where there is high diffusion directionality, usually associated with white matter. It also serves as an effective termination criteria eliminating tracts entering CSF where the FA is low. Parameters like tract angle and tract length thresholding can be adjusted to benefit specific structures (http://trackvis.org/ blog/tag/diffusion-toolkit/)., ${ }^{2,7}$

Two FA thresholds were used for TD and SCI patients to preserve a sufficiently high FA value to be able to initialize fiber tracking at seed point. Also, as diffusion characteristics were different for controls and patients with SCI, the FA threshold was defined individually and set to $40-50 \%$ below the mean FA value. ${ }^{18,19}$ The lower FA thresholds for all TD and SCI patients were set to 0.30 and 0.15 , respectively. The fiber tracts generated were constrained within the limits of these thresholds as well as when the fiber track turns by more than a particular angle threshold, in this case $70^{\circ}$. Also, a lower tract length threshold of $4.8 \mathrm{~mm}$ was set to eliminate fiber fragments caused by noise within the tract reconstruction.

For each subject, a manually drawn ROI was defined at every axial slice along the entire spinal cord to create a single, conglomerate and three-dimensional ROI that completely encompassed the tracts of the spinal cord, as shown in Figure 1. Because of the intersubject variability in terms of cord volume, ROIs were subject specific. What remained consistent was that ROIs cover the entire cord from $\mathrm{C} 1$ to $\mathrm{C} 7$. For each case, statistical values for tract length, FA and ADC were obtained. In addition, tract density was also measured, on the basis of the FACT algorithm. The mean length and density of the fiber tracts derived from tracks that are only lying within the ROI were reported.

\section{Statistics}

Upon definition of whole cord ROIs, statistical analysis was performed between patient and healthy groups. A comprehensive data table was created containing information for FA, ADC, tract length and tract density for each subject (Tables 2 and 3). The mean and standard deviation of the DTI and DTT indices for every subject along the cervical spinal cord were calculated. Using mean and standard deviation, the unpaired $t$-test was performed to determine the statistical significance of DTI and DTT indices between SCI and controls. 
Table 2 DTI and DTT parameters obtained in control subjects

\begin{tabular}{lclcc}
\hline Subject & Mean FA & Mean $A D C\left(\mathrm{~mm}^{2} \mathrm{~s}^{-1}\right)$ & $\begin{array}{c}\text { Mean length of } \\
\text { tract }(\mathrm{mm})\end{array}$ & Tract density \\
\hline TD1 & $0.58 \pm 0.11$ & $0.902 \pm 0.172 \times 10^{-3}$ & $33.41 \pm 20.46$ & 1350 \\
TD2 & $0.62 \pm 0.12$ & $0.956 \pm 0.175 \times 10^{-3}$ & $20.93 \pm 14.02$ & 825 \\
TD3 & $0.64 \pm 0.14$ & $0.814 \pm 0.205 \times 10^{-3}$ & $22.60 \pm 14.58$ & 1035 \\
TD4 & $0.59 \pm 0.13$ & $0.803 \pm 0.211 \times 10^{-3}$ & $25.01 \pm 17.16$ & 1252 \\
TD5 & $0.58 \pm 0.13$ & $0.560 \pm 0.156 \times 10^{-3}$ & $20.45 \pm 12.83$ & 1218 \\
TD6 & $0.60 \pm 0.13$ & $0.538 \pm 0.152 \times 10^{-3}$ & $24.75 \pm 17.61$ & 1355 \\
TD7 & $0.56 \pm 0.12$ & $0.692 \pm 0.179 \times 10^{-3}$ & $26.63 \pm 19.47$ & 942 \\
TD8 & $0.66 \pm 0.13$ & $0.773 \pm 0.208 \times 10^{-3}$ & $27.85 \pm 17.65$ & 1291 \\
TD9 & $0.57 \pm 0.13$ & $0.531 \pm 0.159 \times 10^{-3}$ & $18.90 \pm 12.07$ & 1272 \\
TD10 & $0.61 \pm 0.12$ & $0.811 \pm 0.190 \times 10^{-3}$ & $22.47 \pm 12.67$ & 1028 \\
Grand & $0.60 \pm 0.13$ & $0.738 \pm 0.181 \times 10^{-3}$ & $24.30 \pm 15.85$ & $1157 \pm 156.1$ \\
mean & & & & \\
\hline
\end{tabular}

Abbreviations: $A D C$, apparent diffusion coefficient; FA, fractional anisotropy.

Table 3 DTI and DTT parameters obtained in SCI subjects

\begin{tabular}{llllr}
\hline Subject & Mean FA & Mean ADC $\left(\mathrm{mm}^{2} \mathrm{~s}^{-1}\right)$ & $\begin{array}{c}\text { Mean length of } \\
\text { tract }(\mathrm{mm})\end{array}$ & $\begin{array}{r}\text { Tract } \\
\text { density }\end{array}$ \\
\hline SCI1 & $0.52 \pm 0.16$ & $0.682 \pm 0.220 \times 10^{-3}$ & $30.84 \pm 20.28$ & 848 \\
SCl2 & $0.42 \pm 0.13$ & $0.965 \pm 0.244 \times 10^{-3}$ & $19.83 \pm 17.13$ & 894 \\
SCI3 & $0.49 \pm 0.16$ & $0.907 \pm 0.355 \times 10^{-3}$ & $17.75 \pm 9.12$ & 684 \\
SCI4 & $0.38 \pm 0.14$ & $0.829 \pm 0.267 \times 10^{-3}$ & $24.06 \pm 15.12$ & 692 \\
SCI5 & $0.56 \pm 0.15$ & $0.843 \pm 0.272 \times 10^{-3}$ & $29.42 \pm 19.31$ & 1085 \\
SCI6 & $0.38 \pm 0.12$ & $0.922 \pm 0.296 \times 10^{-3}$ & $29.51 \pm 18.31$ & 734 \\
SCI7 & $0.44 \pm 0.14$ & $0.638 \pm 0.186 \times 10^{-3}$ & $25.94 \pm 17.81$ & 818 \\
SCI8 & $0.36 \pm 0.10$ & $0.657 \pm 0.169 \times 10^{-3}$ & $11.56 \pm 4.90$ & 100 \\
SCI9 & $0.41 \pm 0.15$ & $0.674 \pm 0.179 \times 10^{-3}$ & $21.41 \pm 14.93$ & 732 \\
SCI10 & $0.50 \pm 0.16$ & $0.555 \pm 0.180 \times 10^{-3}$ & $20.25 \pm 14.20$ & 916 \\
\hline
\end{tabular}

Abbreviations: ADC, apparent diffusion coefficient; FA, fractional anisotropy.

Also, coefficient of variation $(\mathrm{CoV})$ was used to measure the relative variability ${ }^{20}$ of FA, ADC and tract length and to ascertain whether they are consistent within controls and SCI subjects.

\section{RESULTS}

DTT tracts were successfully generated for all cases. Although one can measure peripheral nerves and nerve root white matter fiber bundles using diffusion imaging, our technique used an rFOV acquisition scheme to improve SNR in the cord. We did not focus on imaging the nerve root bundles in this study. However, it is possible to generate not only the axonal bundles of the spinal cord but also the outgoing dorsal and ventral nerve roots with special angle adjustment (for example, $\left.45^{\circ}\right) .{ }^{10}$

Figures 2 and 3 show tractography images of the entire pediatric cervical spinal cord of a normal subject and a patient with SCI, respectively. The tractography images visually showed that the white matter axonal tracts (blue color) of the SCI patients were overall less abundant and less organized as compared with the control cases. Some SCI cases showed a discontinuation of tracts that clearly displayed severe damage to the spinal cord as is evident in Figure 3.

Tables 2 and 3 show the various DTI and DTT parameters obtained from all the normal subjects and patients with SCI. The mean FA values in the controls and patients were $0.6 \pm 0.13$ and $0.45 \pm 0.14$, respectively. FA values were significantly decreased in patients with SCI $(P=0.0238)$ when compared with controls. ADC values in the controls and patients were $0.738 \pm 0.181 \times 10^{-3} \mathrm{~mm}^{2} \mathrm{~s}^{-1}$ and
$0.767 \pm 0.237 \times 10^{-3} \mathrm{~mm}^{2} \mathrm{~s}^{-1}$, respectively; however, it was not a statistically significant difference. These FA and ADC values in controls and patients with SCI were comparable to what is published in the current literature. ${ }^{8-12}$ Also, DTT parameters such as mean length of tracts and tract density were calculated using the FACT algorithm. The mean tract density in the controls and patients were $1157 \pm 156.1$ and $750 \pm 259.4$, respectively, which were significantly decreased in the SCI group $(P=0.0005)$ (Figure 4). However, the mean length of tracts $(24.30 \pm 15.85 \mathrm{~mm}$ and $23.06 \pm 15.11 \mathrm{~mm}$ in controls and patients, respectively) did not show significant differences.

The consistency of the DTI and DTT values was measured using $\mathrm{CoV}$ within the control and SCI groups as mentioned in the statistical methodology section. The CoV of the DTI and DTT values within the control subjects showed FA $(\mathrm{CoV}=0.215 \pm 0.014)$, ADC $(\mathrm{CoV}=0.261 \pm 0.038)$ and tract length $(\mathrm{CoV}=0.641 \pm 0.049)$. Subjects with SCI showed less consistency when compared with controls: $\mathrm{FA}(\mathrm{CoV}=0.317 \pm 0.032), \mathrm{ADC}(\mathrm{CoV}=0.322 \pm 0.042)$ and tract length $(\mathrm{CoV}=0.657 \pm 0.117)$.

\section{DISCUSSION}

Previously DTI and DTT methods were poorly explored in evaluation of the pediatric spinal cord because of the relatively small size of the spinal cord, and the motion artifact induced by CSF pulsation, cardiac and respiration. ${ }^{3,5}$ However, development of newer pulse sequence methods such as reduced FOV has enabled reliable DTI collection and enabled further exploration of spinal cord tracts using DTT. In this study, DTI and deterministic tractography were used to successfully reconstruct white matter fibers in both controls and SCI patients. The results demonstrate that spinal cord DTI and DTT can clearly show displacement and deformation of the white matter tract at the pathological level of the spinal cord.

FA and ADC are widely used parameters for quantification of DTI, and many studies have described both FA and ADC as independent and sensitive predictors of the extent of neuronal damage in spinal cord injury. FA values are generally decreased in the presence of extracellular edema, or where a reduced number of fibers result in increased extracellular space and $\mathrm{ADC}$ values might be useful in monitoring the progression of recovery from SCI (http://trackvis.org/ blog/tag/diffusion-toolkit/). ${ }^{14}$

Previous studies on adult subjects show that FA values of injured cords were much lower than those of uninjured normal cord and ADC values do not differ between abnormal and normal cords in patients and controls (http://trackvis.org/blog/tag/diffusion-toolkit/).,3,10 The current study on the pediatric population shows a similar trend. The FA values were significantly lower in patients, especially in patients with complete injury, compared with controls $(P=0.0238)$.

The ADC values of patients with injury were higher than control values, although not reaching statistical significance. This might be due to the averaging effect of the eigenvalues and perhaps might show significant differences if we separate the contribution of axial and radial diffusivities. As the focus of this paper was to evaluate the major DTT differences between patients and normal subjects, we restricted our reporting to $\mathrm{ADC}$ and $\mathrm{FA}$.

The mean FA and ADC values of the pediatric cervical spinal cord observed in this study are different from the values quoted in the literature for the adult population (http://trackvis.org/blog/tag/ diffusion-toolkit/). $3,4,10$ The difference in the values may be primarily due to the age-related biologic developments associated with white matter maturation, namely axonal growth and myelination. ${ }^{14,21,22}$ In 

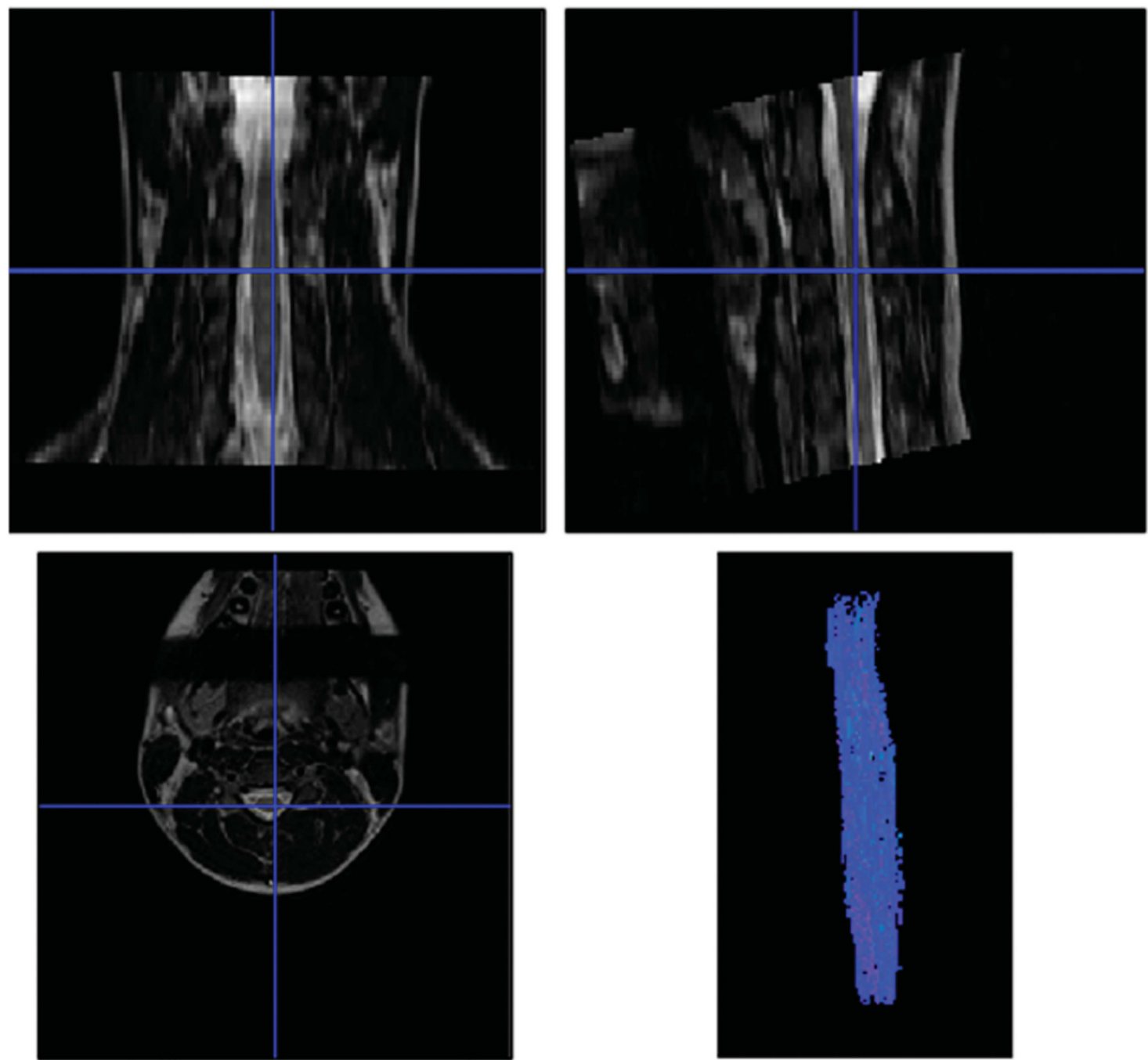

Figure 2 T2- and DTT acquired of the entire cervical spinal cord of a healthy subject. T2-weighted axial (lower left) view, along with reconstructed coronal (upper left) and sagittal (upper right) views, and diffusion tractography of the entire cervical spinal cord (lower right).

neuronal tissue, the orientation of fiber bundles and the axonal diameter, density and myelination have an effect on DTI metrics. ${ }^{22}$

DTT is an important technique that can be used to demonstrate three-dimensional structures of white matter tracts in the brain and spinal cord. DTT was used to identify fiber interruptions in the patients with spinal cord injury. The tractography images visually showed that the white matter tracts (blue color) of the SCI patients were overall less abundant and less organized compared with the control cases. This information could prove clinically relevant, particularly among neurosurgeons during their surgical management of SCI patients. Quantitative measurements such as length and number of fibers along the spinal cord determined by tractography were calculated to evaluate and differentiate SCI patients from healthy subjects as this might provide additional information about the status of the cord. As expected, significant differences were seen in tract density between healthy individuals and SCI subjects (Figure 4). The present data suggest that DTI and DTT indices in the pediatric spinal cord may be effective for delineating the status of damage to the white matter tracts in patients with SCI.

High-resolution rFOV axial DTI images can generate fiber tracts consistent with well-known cord anatomy. In this study, it was difficult to position the ROIs to ensure that all voxels contained white matter, as the area of the spinal cord is small in relation to the large voxel size $\left(1.2 \times 1.2 \times 3 \mathrm{~mm}^{3}\right)$. Therefore, DTI and DTT parameters would be best obtained by excluding gray matter structure by positioning four angularly equidistant ROIs (Deo's procedure) (http://trackvis.org/blog/tag/diffusion-toolkit/). Also, these parameters are sensitive to the quality of DTI raw data and are affected in the presence of noise and subject motion. Therefore, better accuracies can be expected if the DTI images are filtered, voxel size is reduced, or cardiac gating is used. The lowest cervical levels (C4-C7) are the most sensitive to cardiac motion. Therefore, some cardiac-related artifacts may have biased the quantification of DTI and DTT parameters. Although this may be considered a limitation in this study, it is important to note that gating increases the acquisition time, which is not desirable in pediatric imaging. In this study, post-processing using the Automated-Image-Registration package implemented in DTIStudio software was applied to reduce the amount of induced motion artifacts. Also, by restricting the acquisition plane using $\mathrm{rFOV}$ sequence we are reducing the potential effects of motion-induced artifacts as well.

It would also be interesting to compare other DTT algorithms in the future. Deterministic tractography uses the principle of direction of 

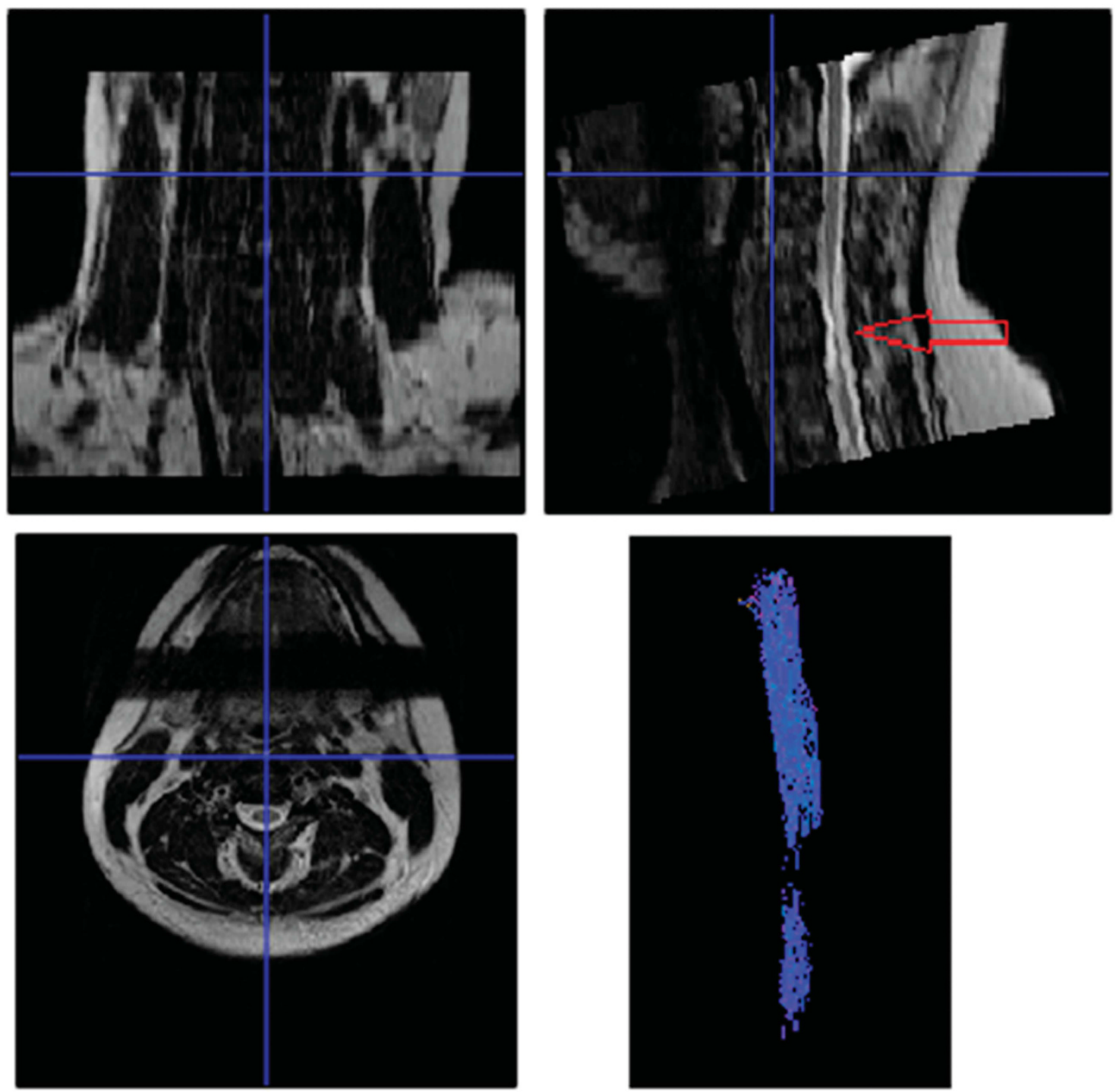

Figure 3 T2- and DTT of a SCl subject acquired of the entire cervical spinal cord. T2-weighted axial (lower left) along with reconstructed coronal (upper left) and sagittal (upper right) views and diffusion tractography of the entire cervical spinal cord (lower right). Three-dimensional tractography shows the disrupted fiber tracts of the cervical spinal cord exactly at the level of injury shown by arrow in the T2-weighted image.

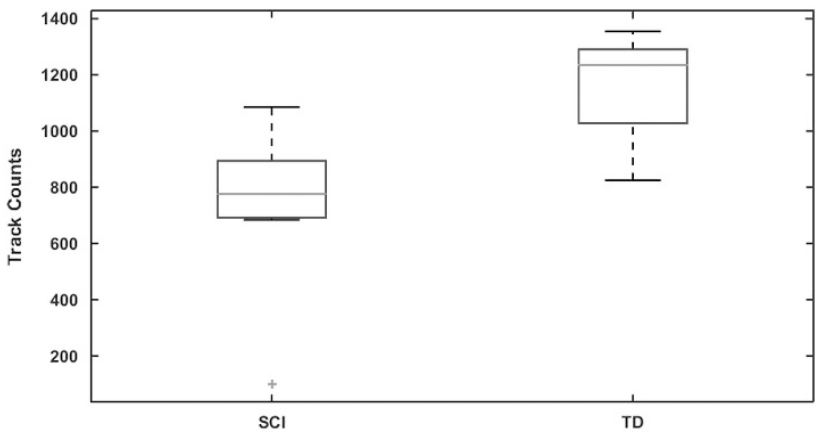

Figure 4 Plot of tract density in TD and SCl subjects. Statistically significant difference is shown in the number of fiber tracks $(P=0.0005)$ between the TD and the $\mathrm{SCl}$ population. A full color version of this figure is available at the Spinal Cord journal online. diffusion to delineate the whole-fiber trajectory. Therefore, it cannot reliably estimate and interpret the crossing-branching fibers. This is the most important limitation of deterministic tractography. ${ }^{12,23}$ Unlike deterministic fiber tracking, probabilistic fiber tracking utilizes the probabilistic distribution of fiber orientations to obtain a set of trajectories, including crossing, branching and main fibers (axonal bundles) connecting to a voxel..$^{23,24}$

It is also worth noting that the relatively small sample size of the current study limits the substantial and consistent significant effects observed and prevents comparison of specific injury sites relative to control subjects. However, the significant $P$ values shown in the comparison of healthy individuals with patients in terms of FA and number of fiber tracts show good evidence and warrant a larger study with various groups of SCI patients. Also, CoV for both healthy and SCI patients is consistent between groups, which supports (though 
does not prove) the assumption of a reasonably good sample from each group.

\section{CONCLUSION}

We have shown that DTT images can be reliably generated from reduced FOV DTI data obtained from the pediatric population using FACT algorithm. The results show a reduction in mean FA as well as in track count for SCI data sets compared with control data sets. In conclusion, these results show that DTI and DTT could be used as a surrogate marker for quantification and visualization of the injured spinal cord and have the potential to improve our understanding of damage and recovery in diseased states of the spinal cord.

\section{DATA ARCHIVING}

There were no data to deposit.

\section{CONFLICT OF INTEREST}

The authors declare no conflict of interest.

\section{ACKNOWLEDGEMENTS}

This work was supported by National Institute of Neurological Disorders of the National Institutes of Health under award number R01NS079635.

1 Fujiyoshi K, Yamada M, Nakamura M, Yamane J, Katoh H, Kitamura K et al. In vivo tracing of neural tracts in the intact and injured spinal cord of marmosets by diffusion tensor tractography. J Neurosci 2007; 27: 11991-11998.

2 Fujiyoshi K, Konomi T, Yamada M, Hikishima K, Tsuji O, Komaki Y et al. Diffusion tensor imaging and tractography of the spinal cord: from experimental studies to clinical application. Exp Neurol 2013; 242: 74-82.

3 Mohamed FB, Hunter LN, Barakat N, Liu C-SJ, Sair H, Samdani AF et al. Diffusion tensor imaging of the pediatric spinal cord at $1.5 \mathrm{~T}$ : preliminary results. AJNR Am J Neuroradiol 2011; 32: 339-345.

4 Mulcahey MJ, Samdani AF, Gaughan JP, Barakat N, Faro S, Shah P et al. Diagnostic accuracy of diffusion tensor imaging for pediatric cervical spinal cord injury. Spinal Cord 2013; 51: 532-537.

5 Barakat N, Mohamed FB, Hunter LN, Shah P, Faro SH, Samdani AF et al. Diffusion tensor imaging of the normal pediatric spinal cord using an inner-FoV EPI sequence. AJNR Am J Neuroradiol 2012; 33: 1127-1133.

6 Middleton DM, Mohamed FB, Barakat N, Hunter LN, Shellikeri S, Finsterbusch J et al. An investigation of motion correction algorithms for pediatric spinal cord DTI in healthy subjects and patients with spinal cord injury. Magn Reson Imaging 2014; 32: 433-439.

7 Mori S, van Zijl PCM. Fiber tracking: principles and strategies-a technical review. NMR Biomed 2002; 15: 468-480.

8 Donald J, Hagler ME Jr, Ahmadi J, Kuperman D, Holland CR, McDonald E et al. Automated white-matter tractography using a probabilistic diffusion tensor atlas: application to temporal lobe epilepsy. Hum Brain Mapp 2009; 30: 1535-1547.

9 Zhixi Li, Peck KK, Brennan1 NP, Jenabi M, Hsu M, Zhang Z et al. Diffusion tensor tractography of the arcuate fasciculus in patients with brain tumors: comparison between deterministic and probabilistic models. J Biomed Sci Eng 2013; 6: 192-200.

10 Mulcahey MJ, Gaughan J, Betz RR, Johansen. KJ. The International Standards for Neurological Classification of spinal cord injury: reliability of data when applied to children and youths. Spinal Cord 2007; 45: 452-459.

11 Tang L, Wen Y, Zhou Z, von Deneen KM, Huang D, Lin Ma. Reduced field-of-view DTI segmentation of cervical spine tissue. Magn Reson Imaging 2013; 31, 1507-1514.

12 Yeh F-C, Verstynen TD, Wang Y, Fernández-Miranda JC, Tseng W-YI. Deterministic diffusion fiber tracking improved by quantitative anisotropy. PLOS ONE 2013; 8: 1-16.

13 Chang Y, Jung TD, Yoo DS, Hyun JK. Diffusion tensor imaging and fiber tractography of patients with cervical spinal cord injury. J Neurotrauma 2010; 27: 2033-2040.

14 Rajasekaran S, Kanna RM, Karunanithi R, Shetty AP. Diffusion tensor tractography demonstration of partially injured spinal cord tracts in a patient with posttraumatic brown sequard syndrome. J Magn Reson Imaging 2010; 32: 978-981.

15 Hobert MK, Stein VM, Dziallas P, Ludwig DC, Tipold A. Evaluation of normal appearing spinal cord by diffusion tensor imaging, fiber tracking, fractional anisotropy, and apparent diffusion coefficient measurement in 13 dogs. Acta Vet Scand 2013; 55: 36.

16 Conklin CJ, Middleton DM, Alizadeh M, Finsterbusch J, Raunig DL, Faro SH et al. Spatially selective 2D RF inner field of view (iFOV) diffusion kurtosis imaging (DKI) of the pediatric spinal cord. Neurolmage Clin 2016; 11: 61-67.

17 Figley CR, Stroman PW. Investigation of human cervical and upper thoracic spinal cord motion: implications for imaging spinal cord structure and function. Magn Reson Med 2007; 58: 185-9.

18 Richter M, Zolal A, Ganslandt O, Buchfelder M, Nimsky C, Merhof D. Evaluation of diffusion-tensor imaging-based global search and tractography for tumor surgery close to the language system. PLOS ONE 2013; 8: 1-14.

19 Knosche TR, Anwander A, Liptrot M, Dyrby TB. Validation of tractography: comparison with manganese tracing. Hum Brain Mapp 2015; 36: 4116-4134.

20 Xu J, Shimony JS, Klawiter EC, Snyder AZ, Trinkaus K, Naismith RT et al. Improved in vivo diffusion tensor imaging of human cervical spinal cord. Neuroimage 2013; 67: 64-76.

21 Gao W, Lin W, Chen Y, Gerig G, Smith JK, Jewells V et al. Temporal and spatial development of axonal maturation and myelination of white matter in the developing brain. AJNR Am J Neuroradiol 2009; 30: 290-96.

22 Koskinen 1 EA, Hakulinen U, Brander AE, Luoto TM, Ylinen A, Ohman JE. Clinical correlates of cerebral diffusion tensor imaging findings in chronic traumatic spinal cord injury. Spinal Cord 2014; 52: 202-208.

23 Abhinav K, Yeh FC, Pathak S, Suski V, Lacomis D, Friedlander RM et al. Advanced diffusion MRI fiber tracking in neurosurgical and neurodegenerative disorders and neuroanatomical studies: a review. Biochim Biophys Acta 2014; 1842: 2286-2297.

24 Cauley KA, Filippi CG. Diffusion-tensor imaging of small nerve bundles: cranial nerves, peripheral nerves, distal spinal cord, and lumbar nerve roots clinical applications. AJR 2013; 201: 326-335. 